\title{
Permeability of human medial collateral ligament in compression transverse to the collagen fiber direction
}

This study quantified the apparent and intrinsic hydraulic permeability of human medial collateral ligament (MCL) under direct permeation transverse to the collagen fiber direction. A custom permeation device was built to apply flow across cylindrical samples of ligament while monitoring the resulting pressure gradient. MCLs from 5 unpaired human knees were used (donor age $55 \pm 16 \mathrm{yr}$, 4 males, 1 female). Permeability measurements were performed at 3 levels of compressive pre-strain $(10 \%, 20 \%$ and $30 \%)$ and 5 pressures $(0.17,0.34,1.03,1.72$ and $2.76 \mathrm{MPa})$. Apparent permeability was determined from Darcy's law, while intrinsic permeability was determined from the zero-pressure crossing of the pressure-permeability curves at each compressive pre-strain. Resulting data were fit to a finite deformation constitutive law [Journal of Biomechanics 23 (1990) 1145-1156]. The apparent permeability of human MCL ranged from $0.40 \pm 0.05$ to $8.60 \pm 0.77 \times 10^{-16} \mathrm{~m}^{4} / \mathrm{N} \mathrm{s}$ depending on pre-strain and pressure gradient. There was a significant decrease in apparent permeability with increasing compressive pre-strain $(p=0.024)$ and pressure gradient $(p<0.001)$, and there was a significant interaction between the effects of compressive pre-strain and pressure $(p<0.001)$. Intrinsic permeability was $14.14 \pm 0.74,6.30 \pm 2.13$ and $4.29 \pm 1.71 \times 10^{-16} \mathrm{~m}^{4} / \mathrm{N}$ s for compressive pre-strains of $10 \%, 20 \%$ and $30 \%$, respectively. The intrinsic permeability showed a faster decrease with increasing compressive pre-strain than that of bovine articular cartilage. These data provide a baseline for investigating the effects of disease and chemical modification on the permeability of ligament and the data should also be useful for modeling the poroelastic material behavior of ligaments. (C) 2004 Elsevier Ltd. All rights reserved.

\section{Introduction}

Ligaments are a biological composite consisting of a ground substance matrix reinforced by collagen and elastin. The ground substance matrix is composed of proteoglycans, glycolipids and fibroblasts and holds large amounts of water, with the total water per wet weight measured as 60-70\% (Chimich et al., 1992; Hey et al., 1990). The water content of ligaments has a strong influence on viscoelastic material properties (Chimich et al., 1992; Thornton et al., 2000). Further, it is believed that water movement in/out and within ligament may play an important role in tissue nutrition, transport of

\footnotetext{
*Corresponding author. Tel.: + 8015877833 ; fax: + 8015855361 .

E-mail address: jeff.weiss@utah.edu (J.A. Weiss).
}

metabolites, mechanotransduction and the overall mechanical properties of the tissue.

A number of explanations have been proposed for the mechanisms governing ligament viscoelasticity. These include viscoelasticity of the collagen fibers (Rubin and Bodner, 2002), the extracellular matrix (Weiss et al., 2002), collagen fibril crosslinking (Bailey et al., 1974; Puxkandl et al., 2002; Redaelli et al., 2003) and fluid content (Chimich et al., 1992) and fluid movement within and in/out of the tissue during loading (Atkinson et al., 1997; Butler et al., 1997). However, most proposals have been based on conjecture and there is little experimental data available to support or refute the proposed mechanisms. Thus, the exact origins of ligament viscoelasticity remain controversial. 
1 Experimental and modeling studies have suggested that the movement of water within and in/out of 3 ligament may partially or entirely explain the viscoelastic response of ligaments (Atkinson et al., 1997; Butler et

5 al., 1997; Chen et al., 1998; Chimich et al., 1992; Hannafin and Arnoczky, 1994). Exudation of water

7 from ligament occurs under cyclic loading (Hannafin and Arnoczky, 1994). Ligament viscoelastic material

9 behavior is strongly coupled to proteoglycan content (Elliott et al., 2003; Thornton et al., 2000; Yamamoto et

11 al., 2000), and elimination of different proteoglycan species increases the amount of relaxation during stress

13 relaxation testing (Elliott et al., 2003). The flow of water through a tissue in response to mechanical or chemical

15 loading is governed by the apparent permeability, sometimes referred to as the hydraulic permeability

17 (Holmes, 1985; Holmes and Mow, 1990; Mansour and Mow, 1976). In the case of ligaments, it has been 19 suggested that the tissue may exhibit anisotropic permeability due to the highly aligned collagen fiber 21 structure (Atkinson et al., 1997; Butler et al., 1997).

Despite the potential importance of fluid movement in ligaments, data on the permeability of ligament to water are not available. Previous computational simulations of ligaments have used ranges of permeability that were motivated primarily by the range of reported values for articular cartilage (Atkinson et al., 1997; Butler et al., 1997). The direct permeation experiment provides a means to determine tissue permeability directly (Holmes, 1985; Holmes and Mow, 1990; Mansour and Mow, 1976). This experiment requires the measurement of flow across a section of tissue in response to an applied pressure gradient under small to moderate levels of compressive strain. Studies of the other hydrated soft tissues under ultrafiltration have demonstrated that both the pressure gradient and the compressive prestrain have a strong influence on the apparent permeability (Gu et al., 2003; Holmes, 1985, 1986; Holmes and Mow, 1990; Lai and Mow, 1980; Lai et al., 1981; Mansour and Mow, 1976; Quinn et al., 2001), and the effects of pressure gradient and compressive pre-strain are coupled (Holmes, 1985; Holmes and Mow, 1990; Lai and Mow, 1980; Lai et al., 1981). The coupling of these effects has been attributed to the nonuniform compaction of the tissue along the direction of permeation, and this effect is magnified with increasing pressure gradient. Although direct measurement of the permeability of ligament along the fiber direction presents technical difficulties due to the fibrous nature of the tissue and the aspect ratio, many ligaments are relatively planar, and it is possible to isolate test specimens from these ligaments that are oriented transverse to the collagen fiber direction for measurement of permeability during ultrafiltration. This mode of testing is relevant to physiological loading of ligaments since tensile loading produces lateral contraction and internal pressure via

the Poisson effect. The objective of this study was to determine the transverse apparent and intrinsic permeability of human medial collateral ligament (MCL) from a direct permeation experiment. Based on the published reports of the apparent permeability of articular cartilage, it was hypothesized that increasing compressive pre-strain and pressure gradients would result in decreases in apparent permeability and that these effects would be coupled.

\section{Materials and methods}

\subsection{Permeation device}

A custom permeation device was designed and built to allow application of small flow rates across cylindrical samples of ligament while monitoring the resulting pressure gradient (Fig. 1). The operation of the device is similar in principle to that described by Gu et al. (1999) in that flow rate is prescribed and the resulting pressure gradient is measured. A syringe pump (SP-101i, WPI, Sarasota, FL) was used with a $500 \mu \mathrm{l}$ glass syringe (Hamilton 1750, Reno, NV) to apply constant flow rates (0.001-100 $\mu \mathrm{l} / \mathrm{min},<1 \%$ error) (Fig. 1, bottom left panel). The area exposed to flow was $3 \mathrm{~mm}$ in diameter. Uniaxial compressive pre-strain was applied to the tissue along the direction of permeation by compressing the top piece of a two-piece acrylic loading fixture against the specimen and an O-ring via a micrometer head (Newport, Irvine, CA, $\pm 1 \mu \mathrm{m}$ accuracy). The O-ring sealed the specimen between the upper and lower pieces of the loading fixture. Relatively rigid (compressive modulus $=18.7 \mathrm{MPa}$ ), highly permeable (hydraulic permeability $=3 \times 10^{-10} \mathrm{~m}^{4} / \mathrm{Ns}$ ) porous polyethylene filters assured free flow to and from the specimen (Porex, Fairburn, GA, $70 \mu \mathrm{m}$ pore size). The resulting pressure gradient was monitored continuously via a pressure transducer (Setra, Boxborough, MA, accuracy $\pm 0.004 \mathrm{MPa})$.

\subsection{Tissue harvest}

The MCL of the human knee was chosen for testing for several reasons. First, the MCL is an extremely important structure in preventing medial joint opening and external tibial rotation in the human knee (Grood et al., 1981; Monahan et al., 1984; Palmer, 1938; Seering et al., 1980; Warren et al., 1974), and approximately $40 \%$ of all severe knee injuries involve the MCL (Miyasaka et al., 1991). Second, because of its planar geometry and size, the MCL is ideal for harvesting transverse samples for direct measurement of permeability. Finally, the results of the present study may compliment existing experimental data on the quasi-static and viscoelastic material properties of the human MCL (Bonifasi-Lista 
et al., 2004; Gardiner and Weiss, 2003; Haridas et al., 2001; Quapp and Weiss, 1998) for future investigations of the origins of ligament viscoelasticity.

Five unpaired human knees (donor age $55 \pm 16 \mathrm{yr}, 4$ males, 1 female) were obtained within $24 \mathrm{~h}$ of death and stored in sealed plastic bags at $-20{ }^{\circ} \mathrm{C}$. On the day of testing, each knee was allowed to thaw at room temperature for $12 \mathrm{~h}$ prior to dissection. All skin, muscle and other periarticular soft tissue surrounding the knee joint were removed. At the time of dissection, no signs of arthritis or previous soft tissue injury were found in any of the knees. The medial side of the knee was fine dissected to expose the superficial MCL. This included removing any adherent fascia by tenting the fascial layer up from the surface of the MCL and dissecting it away gently using a \#15 blade. The MCL was dissected from the knee and placed on a Plexiglas plate. One cylindrical MCL sample (6.5 mm dia.) was punched from each of the 5 human MCL specimens using a hardened steel punch. The thickness of the punched samples was measured using a digital micrometer head combined with a multimeter. The multimeter recorded the resistance between the tip of the micrometer head and a stainless steel plate on which the sample was placed. The micrometer head was lowered toward the surface of the test sample until there was a decrease in resistance between the micrometer head and the plate. The thickness was determined from the micrometer reading at this point. No visible deformation of the tissue was observed when the multimeter recorded the change in resistance. Samples were placed into the lower half of the test chamber and were allowed to equilibrate in Ringer's solution for $1 \mathrm{~h}$ prior to testing. The permeation device was then assembled and sealed, taking care to ensure that all air bubbles were removed from the system before testing.

\subsection{Test protocol}

The test protocol was similar to previous studies of the permeability of articular cartilage (Mansour and Mow, 1976). Permeability measurements were performed at three levels of uniaxial compressive pre-strain $(10 \%, 20 \%$ and $30 \%)$ and 5 pressure gradients of 0.17 , 
$1 \quad 0.34,1.03,1.72$ and $2.76 \mathrm{MPa}(25,50,150,250$ and 400 psi, respectively). First, $10 \%$ pre-strain was applied 3 via the micrometer head. A trial flow rate $(Q)$ was then applied via the syringe pump and the pressure gradient

$5 \Delta P$ was monitored via the pressure transducer. When the pressure gradient reached $0.17 \mathrm{MPa}(25 \mathrm{psi})$, the flow

7 rate was adjusted continuously to maintain the desired pressure gradient. The flow rate was recorded when the

9 variation in pressure gradient was less than $0.01 \mathrm{MPa} / \mathrm{h}$ $(1.5 \mathrm{psi} / \mathrm{h})$. Measurements were repeated at the higher 11 pressure gradients before moving to the next pre-strain. Flow rates during testing were in the range of $0.01-0.3 \mu \mathrm{l} / \mathrm{min}$. The total test time for each sample was approximately $5 \mathrm{~h}$.

\subsection{Apparent permeability}

The apparent permeability $k_{\text {app }}$ was calculated using a one-dimensional version of Darcy's law (Gu et al., 1999; Lai and Mow, 1980):

$k_{\text {app }}=\frac{Q h_{0}(1-\varepsilon)}{A \Delta P}$,

where $Q$ is the volumetric fluid flow rate, $h_{0}$ is the initial thickness of the sample, $\varepsilon$ is the uniaxial compressive pre-strain along the direction of permeation, $A$ is the area exposed to flow, and $\Delta P$ is the pressure gradient across the sample.

\subsection{Intrinsic permeability}

Even in the case when the permeability of the sample is initially homogeneous, a pressure gradient across the sample results in a locally nonuniform compressive strain through the thickness of the sample (Holmes, 1985, 1986; Holmes and Mow, 1990; Lai and Mow, 1980). As the pressure gradient is increased, the compressive strain through the thickness of the sample becomes more nonuniform and thus the local permeability is a function of the local strain. One approach to obtain the intrinsic permeability of the sample from a direct permeation experiment is to extrapolate the apparent permeability versus pressure curves in Fig. 2 to the case of zero applied pressure for each strain level. When the pressure gradient is zero, the resulting data represent the intrinsic permeability of the sample as a function of applied compressive pre-strain only. Based on the appearance of the experimental data and guided by the approach taken by others (Holmes, 1985; Lai and Mow, 1980), the apparent permeability versus pressure data were fit to the following empirical function on a sample-by-sample basis:

$k_{\text {app }}=k_{1}+k_{2} \mathrm{e}^{\left(-M_{1} \Delta P\right)}$.

Here, $k_{1}, k_{2}$ and $M_{1}$ are coefficients that depend on the particular level of compressive strain. In particular, $k_{1}+$

$k_{2}$ represents the apparent permeability for a given strain level at zero pressure gradient, i.e., the intrinsic permeability for a given strain level. Eq. (2) was used to fit the experimental data consisting of pressure gradient and apparent permeability at each level of compressive strain. The coefficients $k_{1}, k_{2}$ and $M_{1}$ in (2) were determined for each sample using a nonlinear leastsquares approach with reciprocal- $y$ weighting. Note that although the function in Eq. (2) provides a good fit to the experimental data, it is nonunique.

To provide data for computational simulations and to allow comparison with published material coefficients for articular cartilage (Ateshian et al., 1997), the experimental data were fit to a constitutive law for intrinsic permeability that was developed for hydrated connective tissues and soft gels (Holmes, 1985, 1986; Holmes and Mow, 1990). The constitutive law has been applied to articular cartilage to describe its intrinsic permeability. Using the average values $\left(k_{1}+k_{2}\right)$ for intrinsic permeability at each strain level, the data were fit to the following permeability constitutive equation with reciprocal- $y$ weighting to determine the associated material coefficients:

$k=k_{0}\left[\frac{\phi_{0} \phi_{\mathrm{f}}}{\left(1-\phi_{0}\right) \phi_{\mathrm{s}}}\right]^{\kappa} \exp \left(M\left(J^{2}-1\right) / 2\right)$.

Here, $J=\operatorname{det}(\boldsymbol{F})$ is the volume ratio and $\boldsymbol{F}$ is the deformation gradient (Spencer, 1980) $(J=1-\varepsilon$ for the permeation experiment), $\phi_{0}$ is the initial volume fraction of the solid phase, taken to be 0.3 for ligament (Atkinson et al., 1997; Butler et al., 1997; Chimich et al., 1992; Hey et al., 1990), $\phi_{\mathrm{f}}=1-\phi_{0} / J$ is the current volume fraction of the fluid phase and $\phi_{\mathrm{s}}=\phi_{0} / J$ is the current volume fraction of the solid phase. $k_{0}$ represents the intrinsic permeability in the absence of strain. $M$ controls the rate of change of permeability with changes in volume ratio, with positive values implying a decrease in permeability with increasing compressive pre-strain during a direct permeation experiment. $\kappa$ is a positive parameter that determines how fast the permeability approaches 0 as the volume fraction of the solid phase approaches $1 . \kappa$ was set to 2.0 for the curve fit to the experimental data so that direct comparisons of material parameters could be made to the results of Ateshian et al. (1997).

\subsection{Reliability of permeability measurements}

The reliability of the permeability measurements was verified by performing pilot tests on bovine articular cartilage harvested from the distal femur and comparing values for apparent permeability to published values in the literature. The harvest, sample preparation and testing followed the procedures in the literature (Mansour and Mow, 1976). A total of 5 samples of bovine articular cartilage were obtained from 3 femurs. The
103 
1 first 3 samples were taken from the same femur and were used to determine the appropriate flow rates needed for 3 the experiments. The last 2 samples were harvested from 2 separate femurs and were used to determine the

5 intrinsic permeability at $15 \%$ and $30 \%$ compressive strain, respectively.

\subsection{Statistical analysis}

A 2-way ANOVA with repeated measures was used to assess the effects of compressive pre-strain and pressure gradient on the apparent permeability, and to assess any significant interaction between the effects of compressive pre-strain and pressure gradient, with significance set at $p \leqslant 0.05$.

\section{Results}

\subsection{Apparent permeability}

Values for the apparent permeability were of the same order of magnitude as those reported for bovine articular cartilage (Ateshian et al., 1997; Lai and Mow, 1980; Mansour and Mow, 1976). The results demonstrated a dramatic decrease in apparent permeability with increases in both pre-strain and pressure gradient (Fig. 2). There was a significant decrease in permeability with increasing pressure gradient $(p<0.001)$. There was also a significant decrease in permeability with increasing pre-strain $(p=0.024)$. There was a significant interaction between pressure gradient and pre-strain $(p<0.001)$. The average thickness of the samples was $0.95 \pm 0.05 \mathrm{~mm}$.

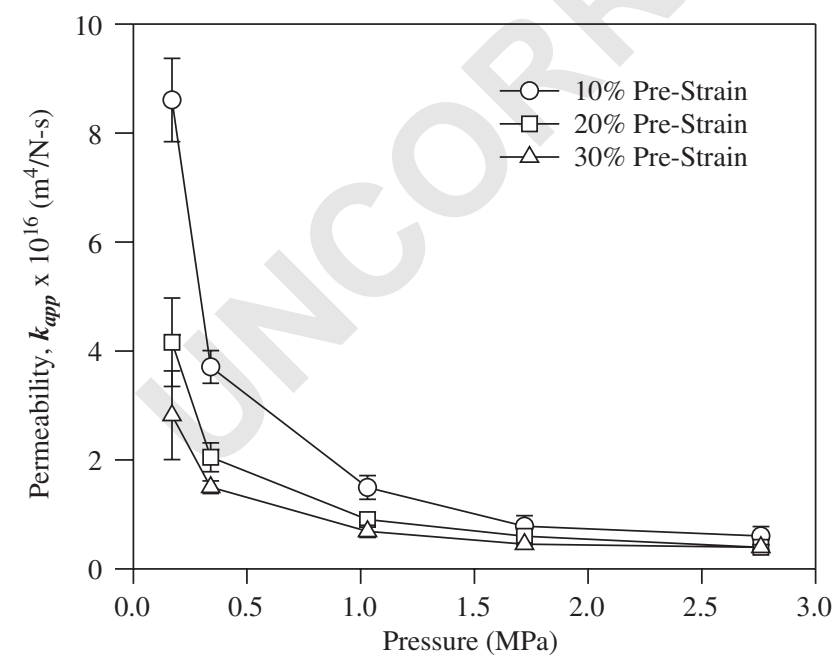

Fig. 2. Transverse apparent permeability of the MCL as a function of pressure gradient and compressive pre-strain (mean \pm standard deviation).

\subsection{Intrinsic permeability}

Using the results for $k_{1}+k_{2}$ obtained for each sample 59 from the curve fit of Eq. (2) at each strain level, calculated values of intrinsic permeability were $14.14 \pm$ $0.74, \quad 6.30 \pm 2.13$ and $4.29 \pm 1.71 \times 10^{-16} \mathrm{~m}^{4} / \mathrm{Ns}$ for compressive strains of $10 \%, 20 \%$ and $30 \%$, respectively. The curve fit of these average data with Eq. (3) provided a good description of the experimental data $\left(k_{0}=29.42 \times 10^{-16} \mathrm{~m}^{4} / \mathrm{N} \mathrm{s}, \quad M=7.988, \quad R^{2}=0.975\right)$ (Fig. 3). Since $M$ was positive, Eq. (3) predicts a decrease in permeability with increasing compressive pre-strain. Compared to intrinsic permeability data from previous studies on bovine articular cartilage (Ateshian et al., 1997; Holmes and Mow, 1990), the permeability of ligament was lower than that of bovine articular cartilage at higher compressive strains but showed a faster increase with decreasing compressive strains. The latter effect is governed by the value of $M$, which was 7.988 for the present study and 2.2 for bovine articular cartilage (Ateshian et al., 1997). The pilot data for the intrinsic permeability of bovine articular cartilage were in reasonable agreement with data from the literature (Fig. 4), with values for apparent permeability at $15 \%$ and $30 \%$ strain that fell between those reported in two other studies (Ateshian et al., 1997; Holmes and Mow, 1990).

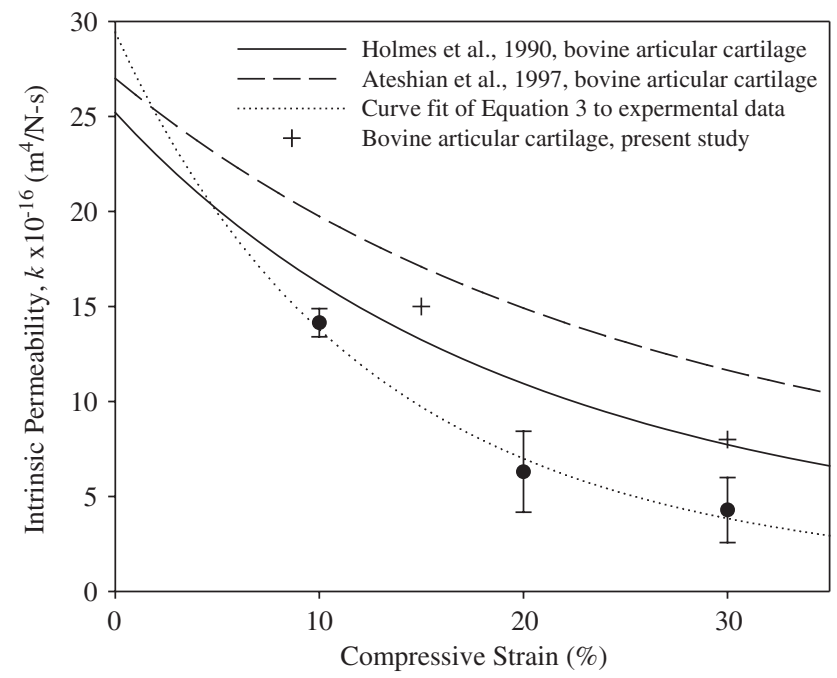

Fig. 3. Intrinsic permeability of the MCL as a function of compressive pre-strain. Circles with error bars indicate experimental data obtained in the present study (mean \pm standard deviation). Solid line indicates fit of Eq. (3) to data $\left(\kappa=2, \phi_{0}=0.3, k_{0}=29.42 \times 10^{-16} \mathrm{~m}^{4} / \mathrm{N} \mathrm{s}, M=\right.$ $7.988, R^{2}=0.975$ ). For comparison, data for bovine articular cartilage are shown from the two pilot tests performed in this study and for two different studies from the literature (Ateshian et al., 1997: $\kappa=2, \phi_{0}=$ $0.2, k_{0}=27.0 \times 10^{-16} \mathrm{~m}^{4} / \mathrm{N} \mathrm{s}, M=2.2$; Holmes and Mow, 1990: $\kappa=$ $\left.0.0848, \phi_{0}=0.2, k_{0}=25.19 \times 10^{-16} \mathrm{~m}^{4} / \mathrm{N} \mathrm{s}, M=4.638\right)$. 


\section{Discussion}

3 The physiological relevance of the range of pressure gradients used in the testing is worthy of discussion.

5 During a uniaxial tensile test of an incompressible material, the sample contracts laterally, resulting in a

7 positive hydrostatic pressure $p$. The pressure is related to the Cauchy stress $\boldsymbol{T}$ by $p=1 / 3 \operatorname{tr}(\boldsymbol{T})$ (Spencer, 1980).

9 Assuming that the direction of uniaxial tensile loading is the 1-direction, the normal stresses $T_{22}$ and $T_{33}$ are zero, 11 which yields $p=T_{11} / 3$. If the material is incompressible, which represents the time-zero behavior of a 13 biphasic material, the pressure can be calculated analytically. Using published material coefficients for 15 human MCL (Gardiner and Weiss, 2003), tensile strains of $1 \%, 3 \%, 5 \%$ and $10 \%$ along the fiber direction result

17 in pressures of $0.26,1.40,4.44$ and $19.00 \mathrm{MPa}$. The pressure gradients used in this study ranged from 0.17 to

$192.76 \mathrm{MPa}$, corresponding to uniaxial tensile strains along the fiber direction of about $0.5-4 \%$. If the 21 material is considered to be even slightly compressible, pressures resulting from tensile loading will be signifi23 cantly lower than for the incompressible case (an analytical solution for the pressure in a compressible 25 material under uniaxial extension is not possible). The natural geometry of ligaments cannot be approximated 27 by a tensile test specimen, and they are subjected to much more complex loading than simply uniaxial 29 extension. These factors will result in pressure gradients inside the ligament during loading in vivo. The 31 magnitude of these pressure gradients will likely be smaller than those resulting from uniaxial extension.

33 Thus the range of pressure gradients examined in this study are physiologically relevant, not only to a large

35 range of uniaxial tensile strains along the fiber direction in the MCL, but also to the more subtle variations in 37 pressure that occur due to nonuniform geometry and boundary conditions in vivo.

39 A second mechanism for the generation of pressure gradients in ligaments under in vivo loading is trans41 verse compression due to wrapping around bones and other ligaments. This is common for ligaments of the 43 knee (Bendjaballah et al., 1997; Li et al., 2002; Sakane et al., 1999), and in particular the MCL (Gardiner and 45 Weiss, 2003). Although our laboratory has performed subject-specific finite element simulations of the human

47 MCL under valgus knee loading (Gardiner and Weiss, 2003), it is impossible to separate the pressure generated

49 by the wrapping of the MCL around the tibia and femur from the pressure that is generated due to tensile stretch 51 along the fiber direction, since the latter stretch is responsible for the wrapping.

53 Compressive transverse strains of $10-30 \%$ were used in this study. The lowest strain was chosen to ensure that the specimen did not separate from the filter. The highest value was chosen so that comparisons could be made to published data for articular cartilage (Mansour and Mow, 1976). Reconsidering the case of uniaxial strain $\varepsilon_{1}$ of an incompressible, transversely isotropic material along the fiber direction, the resulting transverse strain $\varepsilon_{\mathrm{t}}$ will be negative. The deformation gradient $\boldsymbol{F}$ is then

$[\boldsymbol{F}]=\left[\begin{array}{ccc}1+\varepsilon_{\mathrm{l}} & 0 & 0 \\ 0 & 1+\varepsilon_{\mathrm{t}} & 0 \\ 0 & 0 & 1+\varepsilon_{\mathrm{t}}\end{array}\right]$,

and the incompressibility condition $\operatorname{det}(\boldsymbol{F})=1$ yields $\varepsilon_{\mathrm{t}}=\left(1 / \sqrt{1+\varepsilon_{1}}\right)-1$. A tensile strain along the fiber direction as high as $15 \%$ will yield a transverse strain of only $-6.7 \%$. Thus, when considering uniaxial extension along the fiber direction, the permeability values at the lowest strain level of $10 \%$ are the most relevant. Transverse strains resulting from ligament wrapping around bones would be considerably larger than $10 \%$ and thus higher values of compressive strains are relevant for those cases. Overall, the highest pressures and lowest strains in this study are the most relevant cases for in vivo loading.

Measurements for apparent and intrinsic permeability of the MCL are similar in order of magnitude to published data for other biological soft tissues. For comparison, the apparent permeability for human MCL under $30 \%$ compressive pre-strain and $0.17 \mathrm{MPa}(25 \mathrm{psi})$ pressure gradient was $2.82 \pm 0.81 \times 10^{-16} \mathrm{~m}^{4} / \mathrm{N} \mathrm{s}$. The apparent permeability of bovine articular cartilage was approximately $11 \times 10^{-16} \mathrm{~m}^{4} / \mathrm{Ns}$ under the same conditions (Mansour and Mow, 1976). The apparent permeability of human lumbar annulus fibrosus was 11.47 to $19.24 \times 10^{-16} \mathrm{~m}^{4} / \mathrm{N}$ s under $29 \%$ compressive pre-strain and a $0.07 \mathrm{MPa}(10 \mathrm{psi})$ pressure gradient $(\mathrm{Gu}$ et al., 1999). MCL intrinsic permeability showed a faster decrease with increasing compressive pre-strain than has been reported for bovine articular cartilage (Fig. 3). The significant interaction between the effects of pressure gradient and compressive pre-strain is consistent with the reports for articular cartilage (Holmes, 1985; Holmes and Mow, 1990; Lai and Mow, 1980; Lai et al., 1981). When interpreting the above comparisons, it must be emphasized that differences in tissue permeabilities may exist between different species, age groups and genders.

One of the limitations of this study is that only 3 data points were obtained for the intrinsic permeability from the experimental measurements. The reliability of these measurements is considered to be high since they were each determined from 5 measurements of pressure at each level of compressive pre-strain and the standard deviations of the measurements were small. However, testing at additional levels of pre-strain would have provided more data to fit the constitutive model in Eq. (3). Testing at pre-strains less than $10 \%$ presents a 
1 difficulty in that the combination of high pressures and low compressive pre-strains can cause separation of the 3 sample from the filter (Holmes and Mow, 1990). However, the acquisition of apparent permeability data 5 at additional data points above $10 \%$ (e.g., $15 \%$ and $25 \%$ ) would have provided a more reliable estimation of

7 the material coefficients $M$ and $k_{0}$. Nevertheless, the constitutive model and predicted material coefficients

9 for $M$ and $k_{0}$ provide a good description of the experimental data over the range of compressive prestrains that were investigated.

There were several factors related to the samples that

13 were not controlled or investigated in this study. The physiochemical properties of soft tissues and hydrated gels can have a dramatic influence on the permeability (Gu et al., 2003; Iatridis et al., 2003; Maroudas, 1968,

17 1976; Quinn et al., 2001). This includes the concentrations of extracellular matrix proteins such as collagen or

19 agarose in the case of hydrated gels (Gu et al., 2003), proteoglycans (Maroudas, 1968; Quinn et al., 2001) and 21 water content (Gu et al., 2003). Further, no attempt was made to correlate donor age or gender with the 23 measured values for apparent and intrinsic permeability. The effects of these factors on permeability may have 25 important implications for the study of disease, maturation and aging, and should be investigated in future 27 studies. However, the study of the effects of aging and sex on permeability in human knee ligaments will likely 29 require a substantially larger number of samples than were used in this study, especially since donor age cannot be controlled in a study of human knee ligaments. Since donor age and sex were not controlled 33 in this study, they may have influenced the results for permeability. However, the relatively small standard 35 deviations in the data of Figs. 2 and 3 suggest that the effects of these factors were not large for this particular 37 population of samples. Finally, the possible effects of the cross-sectional area that was exposed to flow were 39 not investigated in this study. The size of the area exposed to flow ( $3 \mathrm{~mm}$ dia.) was chosen to be much 41 larger than the diameter of the collagen fibers, yet small enough to ensure that the sample was visibly homo43 geneous with nearly uniform thickness. It is possible that sample diameter could affect results because a larger sample diameter would potentially introduce inhomogeneous tissue and/or tissue with varying thickness,

47 while a smaller sample could potentially violate the continuum assumptions inherent in the analysis due to 49 the physical size of local collagen fibers.

As suggested by other investigators (Atkinson et al., 51 1997; Butler et al., 1997) and supported by measurements of apparent diffusion coefficients in tendons with 53 MRI (Han et al., 2000; Wellen et al., 2004), it is possible that ligament permeability along the collagen fiber the flow of water by the orientation of the extracellular matrix, including collagen fibers and proteoglycans, with respect to the direction of permeation. A relatively new method based on gas magnetic resonance imaging may provide another alternative method, which could potentially quantify the entire permeability tensor without any contact with the tissue (Bencsik and Ramanathan, 2001). Measurements of the apparent diffusion coefficient using MRI also provide an indirect measure of permeability and have been used to quantify the relative ease of water diffusion along the fiber and crossfiber directions in tendon (Han et al., 2000; Wellen et al., 2004).

In summary, this study quantified the apparent and intrinsic permeability of human MCL under compression, transverse to the predominant collagen fiber direction. The apparent permeability showed a significant decrease with both applied pressure gradient and compressive pre-strain, and there was a significant interaction between the effects of pressure gradient and compressive pre-strain. To our knowledge, this is the first report of any direct measurement of permeability in ligaments. In addition to providing baseline data for investigating the effects of disease and chemical modification on the permeability of human ligament, the data will also be useful for modeling studies of the poroelastic material behavior of human ligaments.

\section{Acknowledgements}

Support from NIH Grant \#AR47369 is gratefully acknowledged. We thank Professor Gerard Ateshian for his suggestions regarding the design of the permeation device.

\section{References}

Ateshian, G.A., Warden, W.H., Kim, J.J., Grelsamer, R.P., Mow, V.C., 1997. Finite deformation biphasic material properties of bovine articular cartilage from confined compression experiments. Journal of Biomechanics 30, 1157-1164.

Atkinson, T.S., Haut, R.C., Altiero, N.J., 1997. A poroelastic model that predicts some phenomenological responses of ligaments and tendons. Journal of Biomechanical Engineering 119, 400-405.

Bailey, A.J., Robins, S.P., Balian, G., 1974. Biological significance of the intermolecular crosslinks of collagen. Nature 251, 105-109.

Bencsik, M., Ramanathan, C., 2001. Direct measurement of porous media local hydrodynamical permeability using gas MRI. Magnetic Resonance Imaging 19, 379-383.

Bendjaballah, M.Z., Shirazi-Adl, A., Zukor, D.J., 1997. Finite element analysis of human knee joint in varus-valgus. Clinical Biomechanics (Bristol, Avon) 12, 139-148.

Bonifasi-Lista, C., Lake, S.P., Small, M.S., Weiss, J.A., 2004. Viscoelastic properties of the human medial collateral ligament under longitudinal, transverse and shear loading. Journal of Orthopaedic Research, in press.

Butler, S.L., Kohles, S.S., Thielke, R.J., Chen, C., Vanderby Jr., R., 1997. Interstitial fluid flow in tendons or ligaments: a porous 
medium finite element simulation. Medical \& Biological Engineering \&Computing 35, 742-746.

3 Chen, C.T., Malkus, D.S., Vanderby, R., 1998. A fiber matrix model for interstitial fluid flow and permeability in ligaments and tendons. Biorheology 35, 103-118.

5 Chimich, D., Shrive, N., Frank, C., Marchuk, L., Bray, R., 1992. Water content alters viscoelastic behaviour of the normal adolescent rabbit medial collateral ligament. Journal of Biomechanics 25, 831-837.

Elliott, D.M., Robinson, P.S., Gimbel, J.A., Sarver, J.J., Abboud, J.A., et al., 2003. Effect of altered matrix proteins on quasilinear viscoelastic properties in transgenic mouse tail tendons. Annals of Biomedical Engineering 31, 599-605.

Gardiner, J.C., Weiss, J.A., 2003. Subject-specific finite element analysis of the human medial collateral ligament during valgus knee loading. Journal of Orthopaedic Research 21, 1098-1106.

Grood, E.S., Noyes, F.R., Butler, D.L., Suntay, W.J., 1981. Ligamentous and capsular restraints preventing straight medial and lateral laxity in intact human cadaver knees. Journal of Bone and Joint Surgery (America) 63, 1257-1269.

Gu, W.Y., Mao, X.G., Foster, R.J., Weidenbaum, M., Mow, V.C., Rawlins, B.A., 1999. The anisotropic hydraulic permeability of human lumbar anulus fibrosus. Influence of age, degeneration, direction, and water content. Spine 24, 2449-2455.

Gu, W.Y., Yao, H., Huang, C.Y., Cheung, H.S., 2003. New insight into deformation-dependent hydraulic permeability of gels and cartilage, and dynamic behavior of agarose gels in confined compression. Journal of Biomechanics 36, 593-598.

Han, S., Gemmell, S.J., Helmer, K.G., Grigg, P., Wellen, J.W., et al., 2000. Changes in ADC caused by tensile loading of rabbit achilles tendon: evidence for water transport. Journal of Magnetic Resonance 144, 217-227.

Hannafin, J.A., Arnoczky, S.P., 1994. Effect of cyclic and static tensile loading on water content and solute diffusion in canine flexor tendons: an in vitro study. Journal of Orthopaedic Research 12, 350-356.

Haridas, B., Butler, D.L., Weiss, J.A., 2001. Age dependent differences in compressive viscoelasticity in the rabbit flexor tendon. ASME Summer Bioengineering Conference 50, 105-106.

Hey, N.J., Handley, C.J., Ng, C.K., Oakes, B.W., 1990. Characterization and synthesis of macromolecules by adult collateral ligament. Biochimica ET Biophysica Acta 1034, 73-80.

Holmes, M., 1985. A theoretical analysis for determining the nonlinear hydraulic permeability of a soft tissue from a permeation experiment. Bulletin of Mathematical Biology 47, 669-683.

Holmes, M.H., 1986. Finite deformation of soft tissue: analysis of a mixture model in uni-axial compression. Journal of Biomechanical Engineering 108, 372-381.

Holmes, M.H., Mow, V.C., 1990. The nonlinear characteristics of soft gels and hydrated connective tissues in ultrafiltration. Journal of Biomechanics 23, 1145-1156.

Iatridis, J.C., Laible, J.P., Krag, M.H., 2003. Influence of fixed charge density magnitude and distribution on the intervertebral disc: applications of a poroelastic and chemical electric (PEACE) model. Journal of Biomechanical Engineering 125, 12-24.

Lai, W.M., Mow, V.C., 1980. Drag-induced compression of articular cartilage during a permeation experiment. Biorheology 17, 111-123.

Lai, W.M., Mow, V.C., Roth, V., 1981. Effects of nonlinear straindependent permeability and rate of compression on the stress behavior of articular cartilage. Journal of Biomechanical Engineering 103, 61-66.
Li, G., Suggs, J., Gill, T., 2002. The effect of anterior cruciate ligament injury on knee joint function under a simulated muscle load: a three-dimensional computational simulation. Annals of Biomedical Engineering 30, 713-720.

Mansour, J.M., Mow, V.C., 1976. The permeability of articular cartilage under compressive strain and at high pressures. Journal of Bone and Joint Surgery (America) 58, 509-516.

Maroudas, A., 1968. Physicochemical properties of cartilage in the light of ion exchange theory. Biophysical Journal 8, 575-595.

Maroudas, A., 1976. Transport of solutes through cartilage: permeability to large molecules. Journal of Anatomy 122, 335-347.

Miyasaka, K.C., Daniel, D.M., Stone, M.L., Hirshman, P., 1991. The incidence of knee ligament injuries in the general population. American Journal of Knee Surgery 4, 3-8.

Monahan, J.J., Grigg, P., Pappas, A.M., Leclair, W.J., Marks, T., et al., 1984. In vivo strain patterns in the four major canine knee ligaments. Journal of Orthopaedic Research 2, 408-418.

Palmer, I., 1938. On the injuries to the ligaments of the knee joint. Acta Chirurgica Scandinavica 81, 3-282.

Puxkandl, R., Zizak, I., Paris, O., Keckes, J., Tesch, W., et al., 2002. Viscoelastic properties of collagen: synchrotron radiation investigations and structural model. Philosophical Transaction of the Royal Society of London Series B Biological Sciences 357, 191-197.

Quapp, K.M., Weiss, J.A., 1998. Material characterization of human medial collateral ligament. Journal of Biomechanical Engineering 120, 757-763.

Quinn, T.M., Dierickx, P., Grodzinsky, A.J., 2001. Glycosaminoglycan network geometry may contribute to anisotropic hydraulic permeability in cartilage under compression. Journal of Biomechanics 34, 1483-1490.

Redaelli, A., Vesentini, S., Soncini, M., Vena, P., Mantero, S., Montevecchi, F.M., 2003. Possible role of decorin glycosaminoglycans in fibril to fibril force transfer in relative mature tendons - a computational study from molecular to microstructural level. Journal of Biomechanics 36, 1555-1569.

Rubin, M.B., Bodner, S.R., 2002. A three-dimensional nonlinear model for dissipative response of soft tissue. International Journal of Solids and Structures 39, 5081-5099.

Sakane, M., Livesay, G.A., Fox, R.J., Rudy, T.W., Runco, T.J., Woo, S.L., 1999. Relative contribution of the ACL, MCL, and bony contact to the anterior stability of the knee. Knee Surgery, Sports Traumatology, Arthroscopy: Official Journal of the ESSKA 7, 93-97.

Seering, W.P., Piziali, R.L., Nagel, D.A., Schurman, D.J., 1980. The function of the primary ligaments of the knee in varus-valgus and axial rotation. Journal of Biomechanics 13, 785-794.

Spencer, A.J.M., 1980. Continuum Mechanics. New York.

Thornton, G.M., Leask, G.P., Shrive, N.G., Frank, C.B., 2000. Early medial collateral ligament scars have inferior creep behaviour. Journal of Orthopaedic Research 18, 238-246.

Warren, L.F., Marshall, J.L., Girgis, F., 1974. The prime static stabilizer of the medial side of the knee. Journal of Bone and Joint Surgery (America) 56, 665-674.

Weiss, J.A., Gardiner, J.C., Bonifasi-Lista, C., 2002. Ligament material behavior is nonlinear, viscoelastic and rate-independent under shear loading. Journal of Biomechanics 35, 943-950.

Wellen, J., Helmer, K.G., Grigg, P., Sotak, C.H., 2004. Application of porous-media theory to the investigation of water ADC changes in rabbit Achilles tendon caused by tensile loading. Journal of Magnetic Resonance 170, 49-55.

Yamamoto, E., Hayashi, K., Yamamoto, N., 2000. Effects of stress shielding on the transverse mechanical properties of rabbit patellar tendons. Journal of Biomechanical Engineering 122, 608-614. 